\title{
Kinematic analysis of Stirling engine
}

\author{
Ján Vavro jr. ${ }^{1}$, and Ján Vavro ${ }^{1, *}$ \\ ${ }^{1}$ Faculty of industrial Technologies in Púchov, Alexander Dubček University of Trenčín, I. Krasku \\ 491/30, 02001 Púchov, Slovac Republic
}

\begin{abstract}
The submitted paper presents a kinematic analysis of Stirling engine by the vector method. Position equations are written for various points of the mechanism, and values of velocity and acceleration are obtained from derivation of the equations, depending on time. Equations in matrix form are solved by means of the Matlab program. Graphic dependences of kinematic magnitudes of some points are given in dependence on the angle of rotation of the drive member. Position equations are utilised for the animation of the mechanism. The computational model of the Stirling engine was created in the SolidWorks software program.
\end{abstract}

Keywords: kinematic analysis, planar mechanism, Matlab program, SolidWorks software

\section{Introduction}

In the case of kinematic analysis of planar as well as spatial mechanisms [1-3] and their simulations [4-6], it is possible to use the specific software Matlab program. The advantage of this program is based on the simplicity of programing process, while the obtained results are high accurate in relation to the numerical equation solutions in the whole range of the mechanism movement.

\section{Kinematic analysis of planar mechanism}

Considering the ten-item planar mechanism, the vector method [7] is used for solution relating to field of positions, velocities and accelerations. The numerical solution procedures are carried out for predefined input values (1) if the angular rotation of the drive member (2) is: $\Theta_{1}=q=2.0864 \mathrm{rad}$. The computational model of the Stirling engine is in Fig. 1 and the kinematic scheme can be seen in the Fig. 2. The position equations (3) are derived on the basis of $\phi_{i}(i=1,8)$ variables and by this way, the equations of velocities (4) are obtained and subsequently, the given equations of velocities are transformed to matrix form (15). If the equations (4) are derived again, the equations of accelerations (5) are obtained and then, they are transformed to the matrix form (16). The equations from (6) up

\footnotetext{
*Corresponding author: jan.vavro@fpt.tnuni.sk

Reviewers: Ireneusz Malujda, Kateryna Kravchenko
} 
to (14) are valid and used for calculation of kinematic values of distance, velocity and acceleration for $\mathrm{D}, \mathrm{K}, \mathrm{J}$ points. The given solution procedures were carried out by means of the Matlab program and results of kinematic analysis are shown in Figs. from 3 to 8.

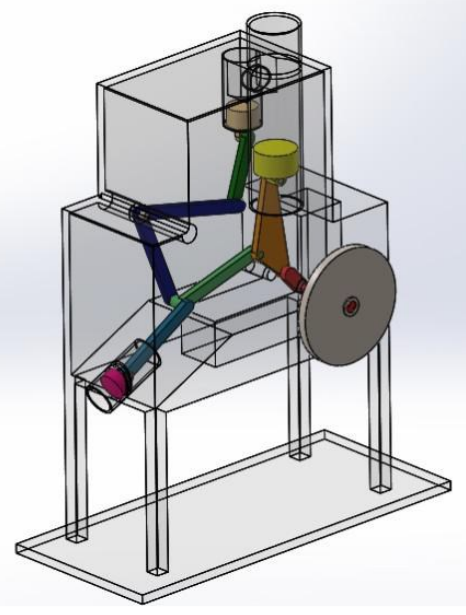

Fig. 1. The computational model of the Stirling engine

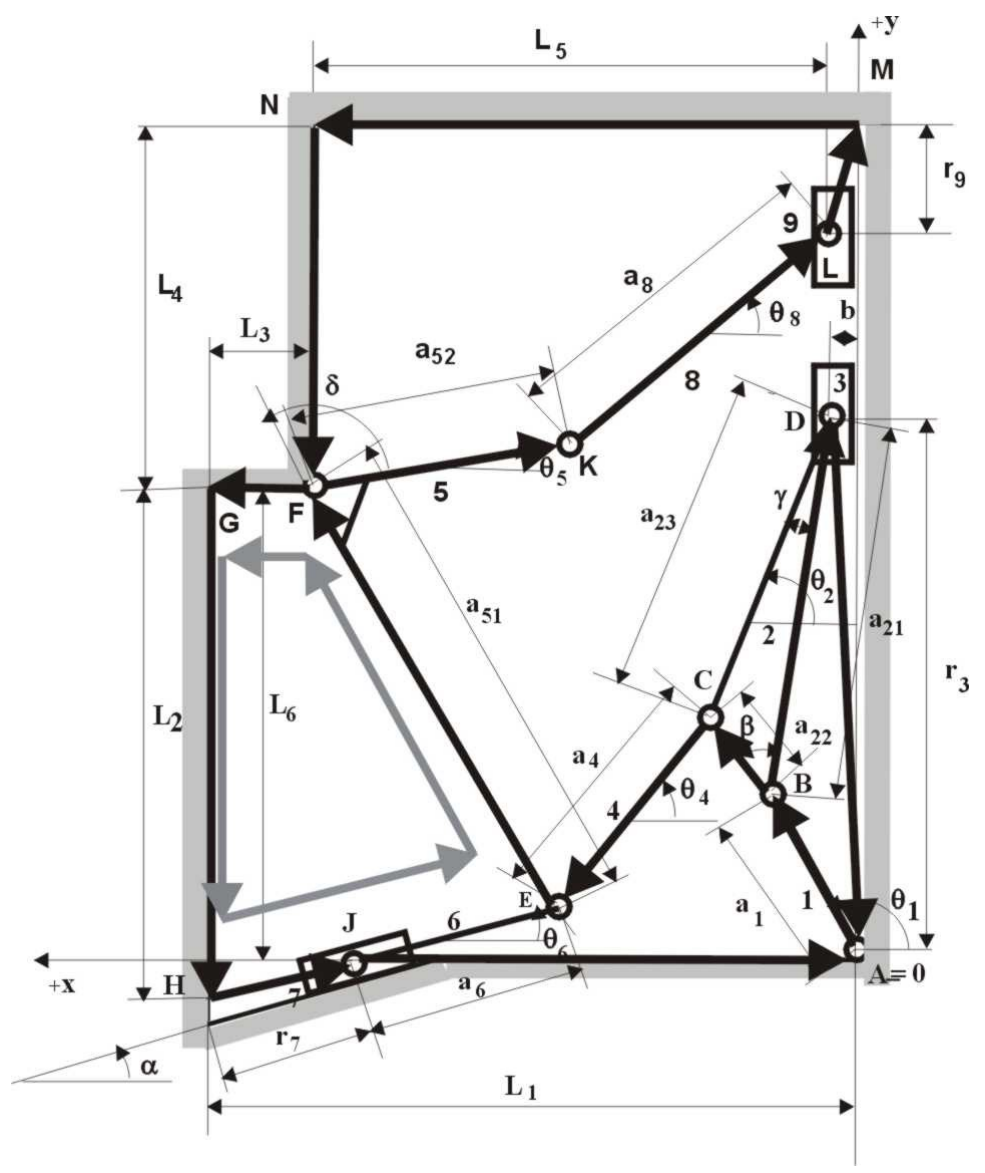

Fig. 2. Kinematic scheme of mechanism 
Input parameters:

$$
\begin{array}{cc}
q=\Theta_{1}=2,0864 \mathrm{rad} & a_{51}=c_{14}=6,0 \mathrm{~cm} \\
\Theta_{2}=\phi_{1}=1,2745 \mathrm{rad} & a_{52}=c_{15}=5,0 \mathrm{~cm} \\
r_{3}=\phi_{2}=7,0 \mathrm{~cm} & a_{6}=c_{16}=2,4 \mathrm{~cm} \\
\Theta_{4}=\phi_{3}=0,3818 \mathrm{rad} & a_{8}=c_{17}=4,8 \mathrm{~cm} \\
\Theta_{5}=\phi_{4}=0,2101 \mathrm{rad} & L_{1}=c_{18}=9,4 \mathrm{~cm} \\
\Theta_{6}=\phi_{5}=0,2003 \mathrm{rad} & L_{2}=c_{19}=6,8 \mathrm{~cm} \\
r_{7}=\phi_{6}=2,4 \mathrm{~cm} & L_{3}=c_{20}=1,9 \mathrm{~cm} \\
\Theta_{8}=\phi_{7}=1,0973 \mathrm{rad} & L_{4}=c_{21}=7,0 \mathrm{~cm} \\
r_{9}=\phi_{8}=1,7 \mathrm{~cm} & L_{5}=c_{22}=7,0 \mathrm{~cm} \\
a_{1}=c_{9}=2,4 \mathrm{~cm} & L_{6}=c_{23}=6,0 \mathrm{~cm} \\
a_{21}=c_{10}=5,0 \mathrm{~cm} & \alpha=c_{24}=0,4907 \mathrm{rad} \\
a_{22}=c_{11}=1,0 \mathrm{~cm} & \beta=c_{25}=0,6338 \mathrm{rad} \\
b=c_{12}=0,4 \mathrm{~cm} & \gamma=c_{26}=1,6258 \mathrm{rad} \\
a_{4}=c_{13}=5,1 \mathrm{~cm} & \delta=c_{27}=0,1411 \mathrm{rad} \\
\omega_{21}=1 \mathrm{rad} . \mathrm{s}^{-1}, & \alpha_{21}=0 \mathrm{rad} . \mathrm{s}^{-2}
\end{array}
$$

Variables are:

$$
\left[\phi_{1}, \phi_{2}, \phi_{3}, \phi_{4}, \phi_{5}, \phi_{6}, \phi_{7}, \phi_{8}\right]=\left[\Theta_{2}, r_{3}, \Theta_{4}, \Theta_{5}, \Theta_{6}, r_{7}, \Theta_{8}, r_{9}\right]
$$

Considering ABDA, ABCEFGHJA, EFGHJE and FKLMNF, the position loop equations are:

$$
\begin{gathered}
f_{1}=-b-a_{21} \cos \left(\phi_{1}-\gamma\right)-a_{1} \cos q=0 \\
f_{2}=-\phi_{2}+a_{21} \sin \left(\phi_{1}-\gamma\right)+a_{1} \sin q=0 \\
f_{3}=-a_{1} \cos q-a_{22} \cos \left(\phi_{1}-\gamma+\beta\right)+a_{4} \cos \phi_{3}-a_{51} \cos \left(\phi_{4}+\delta\right)+L_{3}-L_{1}=0 \\
f_{4}=a_{1} \sin q+a_{22} \sin \left(\phi_{1}-\gamma+\beta\right)-a_{4} \sin \phi_{3}+a_{51} \sin \left(\phi_{4}+\delta\right)-L_{6}=0 \\
f_{5}=-\phi_{6} \cos \alpha-a_{6} \cos \phi_{5}-a_{51} \cos \left(\phi_{4}+\delta\right)+L_{3}=0 \\
f_{6}=\phi_{6} \sin \alpha+a_{6} \sin \phi_{5}+a_{51} \sin \left(\phi_{4}+\delta\right)-L_{2}=0 \\
f_{7}=-a_{52} \cos \phi_{4}-a_{8} \cos \phi_{7}+L_{5}=0 \\
f_{8}=a_{52} \sin \phi_{4}+a_{8} \sin \phi_{7}+\phi_{8}-L_{4}=0
\end{gathered}
$$

When the position equations (3) are derived on the basis of $\phi_{i}(i=1,8)$ variables, the system of velocity equations (4) is obtained and after further derivation, the system of acceleration equations (5) can be obtained.

$$
\begin{aligned}
& \dot{f}_{1}=a_{21} \dot{\phi}_{1} \sin \left(\phi_{1}-\gamma\right)=-a_{1} \dot{q} \sin q \\
& \dot{f}_{2}=-\dot{\phi}_{2}+a_{21} \dot{\phi}_{1} \cos \left(\phi_{1}-\gamma\right)=-a_{1} \dot{q} \cos q
\end{aligned}
$$




$$
\begin{gathered}
\dot{f}_{3}=a_{22} \dot{\phi}_{1} \sin \left(\phi_{1}-\gamma+\beta\right)-a_{4} \dot{\phi}_{3} \sin \phi_{3}+a_{51} \dot{\phi}_{4} \sin \left(\phi_{4}+\delta\right)=-a_{1} \dot{q} \sin q \\
\dot{f}_{4}=a_{22} \dot{\phi}_{1} \cos \left(\phi_{1}-\gamma+\beta\right)-a_{4} \dot{\phi}_{3} \cos \phi_{3}+a_{51} \dot{\phi}_{4} \cos \left(\phi_{4}+\delta\right)=-a_{1} \dot{q} \cos q \\
\dot{f}_{5}=-\dot{\phi}_{6} \cos \alpha+a_{6} \dot{\phi}_{5} \sin \phi_{5}+a_{51} \dot{\phi}_{4} \sin \left(\phi_{4}+\delta\right)=0 \\
\dot{f}_{6}=\dot{\phi}_{6} \sin \alpha+a_{6} \dot{\phi}_{5} \cos \phi_{5}+a_{51} \dot{\phi}_{4} \cos \left(\phi_{4}+\delta\right)=0 \\
\dot{f}_{7}=a_{52} \dot{\phi}_{4} \sin \phi_{4}+a_{8} \dot{\phi}_{7} \sin \phi_{7}=0 \\
\dot{f}_{8}=a_{52} \dot{\phi}_{4} \cos \phi_{4}+a_{8} \dot{\phi}_{7} \cos \phi_{7}+\dot{\phi}_{8}=0
\end{gathered}
$$

$$
\begin{aligned}
& \ddot{f}_{1}=a_{21} \ddot{\phi}_{1} \sin \left(\phi_{1}-\gamma\right)+a_{21} \dot{\phi}_{1}^{2} \cos \left(\phi_{1}-\gamma\right)=-a_{1} \ddot{q} \sin q-a_{1} \dot{q}^{2} \cos q \\
& \ddot{f}_{2}=-\ddot{\phi}_{2}+a_{21} \ddot{\phi}_{1} \cos \left(\phi_{1}-\gamma\right)-a_{21} \dot{\phi}_{1}^{2} \sin \left(\phi_{1}-\gamma\right)=-a_{1} \ddot{q} \cos q+a_{1} \dot{q}^{2} \sin q \\
& \ddot{f}_{3}=a_{22} \ddot{\phi}_{1} \sin \left(\phi_{1}-\gamma+\beta\right)+a_{22} \dot{\phi}_{1}^{2} \cos \left(\phi_{1}-\gamma+\beta\right)-a_{4} \ddot{\phi}_{3} \sin \phi_{3}-a_{4} \dot{\phi}_{3}^{2} \cos \phi_{3}+ \\
& +a_{51} \ddot{\phi}_{4} \sin \left(\phi_{4}+\delta\right)+a_{51} \dot{\phi}_{4}^{2} \cos \left(\phi_{4}+\delta\right)=-a_{1} \ddot{q} \sin q-a_{1} \dot{q}^{2} \cos q \\
& \ddot{f}_{4}=a_{22} \ddot{\phi}_{1} \cos \left(\phi_{1}-\gamma+\beta\right)-a_{22} \dot{\phi}_{1}^{2} \sin \left(\phi_{1}-\gamma+\beta\right)-a_{4} \ddot{\phi}_{3} \cos \phi_{3}+ \\
& +a_{4} \dot{\phi}_{3}^{2} \sin \phi_{3}+a_{51} \ddot{\phi}_{4} \cos \left(\phi_{4}+\delta\right)-a_{51} \dot{\phi}_{4}^{2} \sin \left(\phi_{4}+\delta\right)=-a_{1} \ddot{q} \cos q+a_{1} \dot{q}^{2} \sin q \\
& \ddot{f}_{5}=-\ddot{\phi}_{6} \cos \alpha+a_{6} \ddot{\phi}_{5} \sin \phi_{5}+a_{6} \dot{\phi}_{5}^{2} \cos \phi_{5}+a_{51} \ddot{\phi}_{4} \sin \left(\phi_{4}+\delta\right)+a_{51} \dot{\phi}_{4}^{2} \cos \left(\phi_{4}+\delta\right)=0 \\
& \ddot{f}_{6}=\ddot{\phi}_{6} \sin \alpha+a_{6} \ddot{\phi}_{5} \cos \phi_{5}-a_{6} \dot{\phi}_{5}^{2} \sin \phi_{5}+a_{51} \ddot{\phi}_{4} \cos \left(\phi_{4}+\delta\right)-a_{51} \dot{\phi}_{4}^{2} \sin \left(\phi_{4}+\delta\right)=0 \\
& \ddot{f}_{7}=-a_{52} \ddot{\phi}_{4} \sin \phi_{4}-a_{52} \dot{\phi}_{4}^{2} \cos \phi_{4}-a_{8} \ddot{\phi}_{7} \sin \phi_{7}-a_{8} \dot{\phi}_{7}^{2} \cos \phi_{7}=0 \\
& \ddot{f}_{8}=a_{52} \ddot{\phi}_{4} \cos \phi_{4}-a_{52} \dot{\phi}_{4}^{2} \sin \phi_{4}+a_{8} \ddot{\phi}_{7} \cos \phi_{7}-a_{8} \dot{\phi}_{7}^{2} \sin \phi_{7}+\ddot{\phi}_{8}=0
\end{aligned}
$$

The equations from (6) up to (14) are valid and used for calculation of kinematic values of distance, velocity and acceleration for $\mathrm{D}, \mathrm{K}, \mathrm{J}$ points.

$$
\begin{aligned}
& x_{D}=b \\
& y_{D}=a_{1} \sin q+a_{21} \sin \left(\phi_{1}-\gamma\right)=\phi_{2} \\
& \dot{x}_{D}=0 \\
& \dot{y}_{D}=a_{1} \cdot \dot{q} \cos q+a_{21} \cdot \dot{\phi}_{1} \cos \left(\phi_{1}-\gamma\right)=\dot{\phi}_{2} \\
& \ddot{x}_{D}=0 \\
& \ddot{y}_{D}=a_{1} \cdot \ddot{q} \cos q-a_{1} \cdot \dot{q}^{2} \sin q+a_{21} \ddot{\phi}_{1} \cos \left(\phi_{1}-\gamma\right)-a_{21} \cdot \dot{\phi}^{2}{ }_{1} \sin \left(\phi_{1}-\gamma\right)=\ddot{\phi}_{2} \\
& x_{L}=b \\
& y_{L}=a_{1} \sin q+a_{22} \sin \left(\phi_{1}-\gamma+\beta\right)-a_{4} \sin \phi_{3}+a_{51} \sin \left(\phi_{4}+\delta\right)+a_{52} \sin \phi_{4}+ \\
& +a_{8} \sin \phi_{7}=L_{6}+L_{4}-\phi_{8} \\
& \dot{x}_{L}=0 \\
& \dot{y}_{L}=a_{1} \cdot \dot{q} \cos q+a_{22} \cdot \dot{\phi}_{1} \cos \left(\phi_{1}-\gamma+\beta\right)-a_{4} \cdot \dot{\phi}_{3} \cos \phi_{3}+a_{51} \cdot \dot{\phi}_{4} \cos \left(\phi_{4}+\delta\right)+ \\
& +a_{52} \cdot \dot{\phi}_{4} \cos \phi_{4}+a_{8} \cdot \dot{\phi}_{7} \cos \phi_{7}=-\dot{\phi}_{8}
\end{aligned}
$$




$$
\begin{aligned}
& \ddot{x}_{L}=0 \\
& \ddot{y}_{L}=a_{1} \cdot \ddot{q} \cos q-a_{1} \cdot \dot{q}^{2} \sin q+a_{22} \cdot \ddot{\phi}_{1} \cos \left(\phi_{1}-\gamma+\beta\right)-a_{22} \cdot \ddot{\phi}_{1}^{2} \sin \left(\phi_{1}-\gamma+\beta\right)- \\
& -a_{4} \cdot \ddot{\phi}_{3} \cos \phi_{3}+a_{4} \cdot \dot{\phi}_{3}^{2} \sin \phi_{3}+a_{51} \cdot \ddot{\phi}_{4} \cos \left(\phi_{4}+\delta\right)-a_{51} \cdot \dot{\phi}_{4}^{2} \sin \left(\phi_{4}+\delta\right)+ \\
& +a_{52} \cdot \ddot{\phi}_{4} \cos \phi_{4}-a_{52} \cdot \dot{\phi}_{4}^{2} \sin \phi_{4}+a_{8} \cdot \ddot{\phi}_{7} \cos \phi_{7}-a_{8} \cdot \dot{\phi}_{7}^{2} \sin \phi_{7}=-\ddot{\phi}_{8} \\
& x_{J}=-a_{1} \cos q-a_{22} \cos \left(\phi_{1}-\gamma+\beta\right)+a_{4} \cos \phi_{3}+a_{6} \cos \phi_{5}=L_{1}-\phi_{6} \cos \alpha \\
& y_{J}=a_{1} \sin q+a_{22} \sin \left(\phi_{1}-\gamma+\beta\right)-a_{4} \sin \phi_{3}-a_{6} \sin \phi_{5}=L_{6}-L_{2}+\phi_{6} \cos \alpha \\
& \dot{x}_{J}=a_{1} \cdot \dot{q} \sin q+a_{22} \cdot \dot{\phi}_{1} \sin \left(\phi_{1}-\gamma+\beta\right)-a_{4} \cdot \dot{\phi}_{3} \sin \phi_{3}-a_{6} \cdot \dot{\phi}_{5} \sin \phi_{5}=-\dot{\phi}_{6} \cos \alpha \\
& \dot{y}_{J}=a_{1} \cdot \dot{q} \cos q+a_{22} \cdot \dot{\phi}_{1} \cos \left(\phi_{1}-\gamma+\beta\right)-a_{4} \cdot \dot{\phi}_{3} \cos \phi_{3}-a_{6} \cdot \dot{\phi}_{5} \cos \phi_{5}=\dot{\phi}_{6} \sin \alpha \\
& \ddot{x}_{J}=a_{1} \cdot \ddot{q} \sin q+a_{1} \cdot \dot{q}^{2} \cos q+a_{22} \cdot \ddot{\phi}_{1} \sin \left(\phi_{1}-\gamma+\beta\right)+a_{22} \cdot \dot{\phi}_{1}^{2} \cos \left(\phi_{1}-\gamma+\beta\right)- \\
& -a_{4} \cdot \ddot{\phi}_{3} \sin \phi_{3}-a_{4} \cdot \dot{\phi}_{3}^{2} \cos \phi_{3}-a_{6} \cdot \ddot{\phi}_{5} \sin \phi_{5}-a_{6} \cdot \dot{\phi}_{5}^{2} \cos \phi_{5}=-\ddot{\phi}_{6} \cos \alpha \\
& \ddot{y}_{J}=a_{1} \cdot \ddot{q} \cos q-a_{1} \cdot \dot{q}^{2} \sin q+a_{22} \cdot \ddot{\phi}_{1} \cos \left(\phi_{1}-\gamma+\beta\right)-a_{22} \cdot \dot{\phi}_{1}^{2} \sin \left(\phi_{1}-\gamma+\beta\right)- \\
& -a_{4} \cdot \ddot{\phi}_{3} \cos \phi_{3}+a_{4} \cdot \dot{\phi}_{3}^{2} \sin \phi_{3}-a_{6} \cdot \ddot{\phi}_{5} \cos \phi_{5}+a_{6} \cdot \dot{\phi}_{5}^{2} \sin \phi_{5}=\ddot{\phi}_{6} \sin \alpha
\end{aligned}
$$

The system of equations (4) is transformed to matrix form (15) and the system of equations (5) is transformed to the matrix form (16).

$\left[\begin{array}{cccccccc}a_{21} \sin \left(\phi_{1}-\gamma\right) & 0 & 0 & 0 & 0 & 0 & 0 & 0 \\ a_{21} \cos \left(\phi_{1}-\gamma\right) & -1 & 0 & 0 & 0 & 0 & 0 & 0 \\ a_{22} \sin \left(\phi_{1}-\gamma+\beta\right) & 0 & -a_{4} \sin \phi_{3} & +a_{51} \sin \left(\phi_{4}+\delta\right) & 0 & 0 & 0 & 0 \\ a_{22} \cos \left(\phi_{1}-\gamma+\beta\right) & 0 & -a_{4} \cos \phi_{3} & a_{51} \cos \left(\phi_{4}+\delta\right) & 0 & 0 & 0 & 0 \\ 0 & 0 & 0 & +a_{51} \sin \left(\phi_{4}+\delta\right) & a_{6} \sin \phi_{5} & -\cos \alpha & 0 & 0 \\ 0 & 0 & 0 & a_{51} \cos \left(\phi_{4}+\delta\right) & a_{6} \cos \phi_{5} & \sin \alpha & 0 & 0 \\ 0 & 0 & 0 & a_{52} \sin \phi_{4} & 0 & 0 & a_{8} \sin \phi_{7} & 0 \\ 0 & 0 & 0 & a_{52} \cos \phi_{4} & 0 & 0 & a_{8} \cos \phi_{7} & 1\end{array}\right]$.

$$
\cdot\left[\begin{array}{c}
\dot{\phi}_{1} \\
\dot{\phi}_{2} \\
\dot{\phi}_{3} \\
\dot{\phi}_{4} \\
\dot{\phi}_{5} \\
\dot{\phi}_{6} \\
\dot{\phi}_{7} \\
\dot{\phi}_{8}
\end{array}\right]=\left[\begin{array}{c}
-a_{1} \dot{q} \sin q \\
-a_{1} \dot{q} \cos q \\
-a_{1} \dot{q} \sin q \\
-a_{1} \dot{q} \cos q \\
0 \\
0 \\
0 \\
0
\end{array}\right]
$$




$$
\begin{aligned}
& {\left[\begin{array}{cccccccc}
a_{21} \sin \left(\phi_{1}-\gamma\right) & 0 & 0 & 0 & 0 & 0 & 0 & 0 \\
a_{21} \cos \left(\phi_{1}-\gamma\right) & 1 & 0 & 0 & 0 & 0 & 0 & 0 \\
a_{22} \sin \left(\phi_{1}-\gamma+\beta\right) & 0 & -a_{4} \sin \phi_{3} & a_{51} \sin \left(\phi_{4}+\delta\right) & 0 & 0 & 0 & 0 \\
a_{22} \cos \left(\phi_{1}-\gamma+\beta\right) & 0 & -a_{4} \cos \phi_{3} & a_{51} \cos \left(\phi_{4}+\delta\right) & 0 & 0 & 0 & 0 \\
0 & 0 & 0 & a_{51} \sin \left(\phi_{4}+\delta\right) & a_{6} \sin \phi_{5} & -\cos \alpha & 0 & 0 \\
0 & 0 & 0 & a_{51} \cos \left(\phi_{4}+\delta\right) & a_{6} \cos \phi_{5} & \sin \alpha & 0 & 0 \\
0 & 0 & 0 & a_{52} \sin \phi_{4} & 0 & 0 & a_{8} \sin \phi_{7} & 0 \\
0 & 0 & 0 & a_{52} \cos \phi_{4} & 0 & 0 & a_{8} \cos \phi_{7} & 1
\end{array}\right] \cdot\left[\begin{array}{c}
\ddot{\phi}_{1} \\
\ddot{\phi}_{2} \\
\ddot{\phi}_{3} \\
\ddot{\phi}_{4} \\
\ddot{\phi}_{5} \\
\ddot{\phi}_{6} \\
\ddot{\phi}_{7} \\
\ddot{\phi}_{8}
\end{array}\right]=} \\
& =\left[\begin{array}{c}
-a_{21} \dot{\phi}_{1}^{2} \cos \left(\phi_{1}-\gamma\right)-a_{1} \ddot{q} \sin q-a_{1} \dot{q}^{2} \cos q \\
a_{21} \dot{\phi}_{1}^{2} \sin \left(\phi_{1}-\gamma\right)-a_{1} \ddot{q} \cos q+a_{1} \dot{q}^{2} \sin q \\
a_{22} \dot{\phi}_{1}^{2} \cos \left(\phi_{1}-\gamma+\beta\right)+a_{4} \dot{\phi}_{3}^{2} \cos \phi_{3}-a_{51} \dot{\phi}_{4}^{2} \cos \left(\phi_{4}+\delta\right)-a_{1} \ddot{q} \sin q-a_{1} \dot{q}^{2} \cos q \\
a_{22} \dot{\phi}_{1}^{2} \sin \left(\phi_{1}-\gamma+\beta\right)-a_{4} \dot{\phi}_{3}^{2} \sin \phi_{3}+a_{51} \dot{\phi}_{4}^{2} \sin \left(\phi_{4}+\delta\right)-a_{1} \ddot{q} \cos q+a_{1} \dot{q}^{2} \sin q \\
-a_{6} \dot{\phi}_{5}^{2} \cos \phi_{5}-a_{51} \dot{\phi}_{4}^{2} \cos \left(\phi_{4}+\delta\right) \\
a_{6} \dot{\phi}_{5}^{2} \sin \phi_{5}+a_{51} \dot{\phi}_{4}^{2} \sin \left(\phi_{4}+\delta\right) \\
-a_{52} \dot{\phi}_{4}^{2} \cos \phi_{4}-a_{8} \dot{\phi}_{7}^{2} \cos \phi_{7} \\
a_{52} \dot{\phi}_{4}^{2} \sin \phi_{4}+a_{8} \dot{\phi}_{7}^{2} \sin \phi_{7}
\end{array}\right]
\end{aligned}
$$

\section{Results of kinematic analysis}

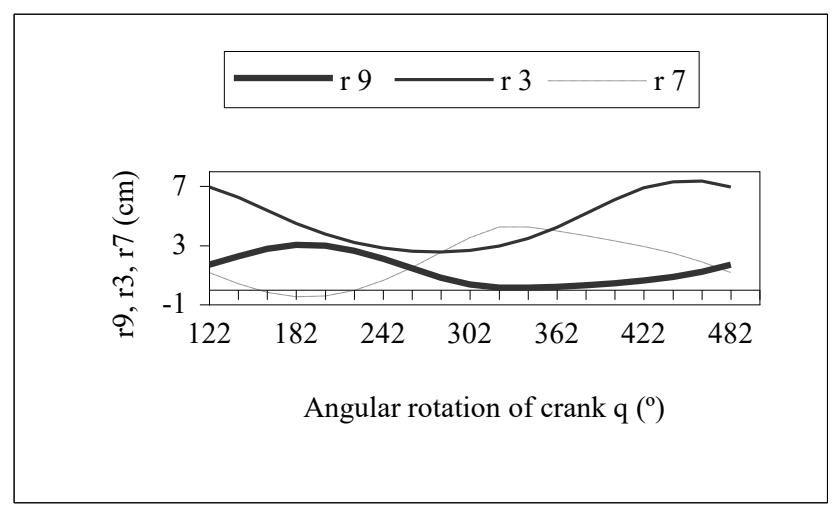

Fig.3. The course of shifts for D, L, J points in dependence on angular rotation of crank

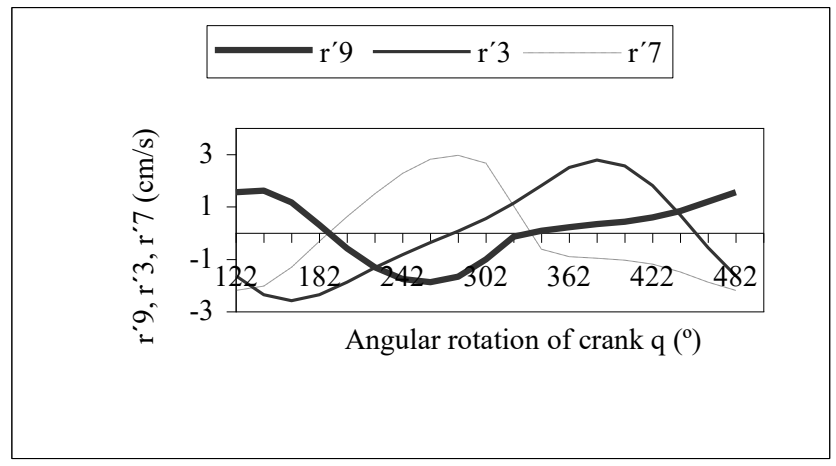

Fig. 4. The course of velocity for D, L, J points in dependence on angular rotation of crank 


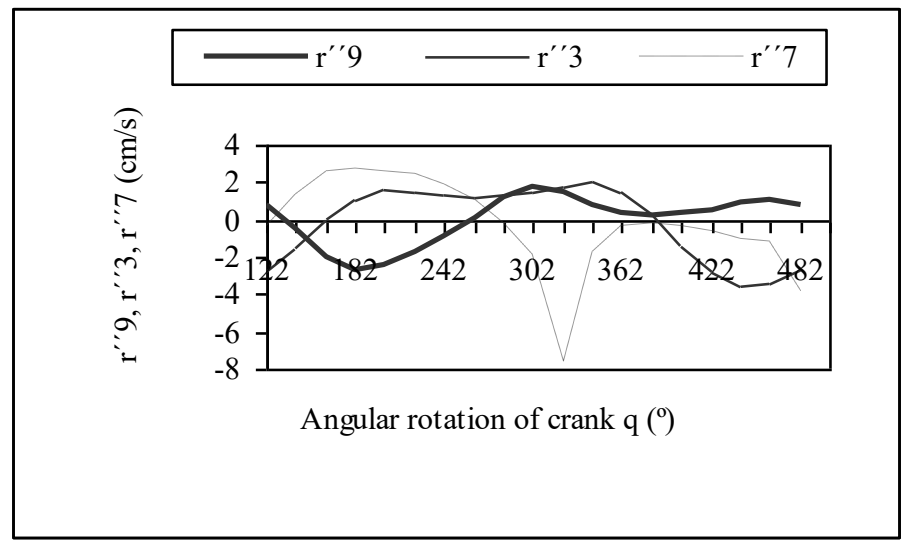

Fig. 5. The course of acceleration for D, L, J points in dependence on angular rotation of crank

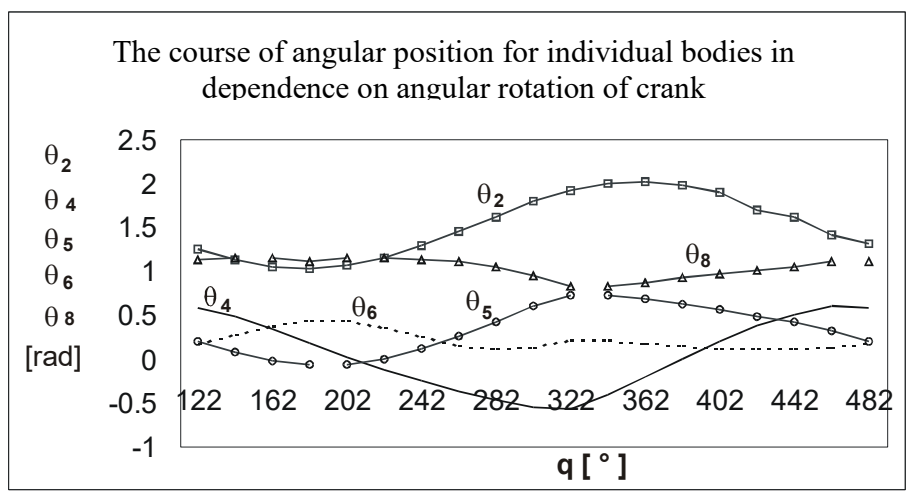

Fig. 6. The course of angular position for individual bodies in dependence on angular rotation of crank

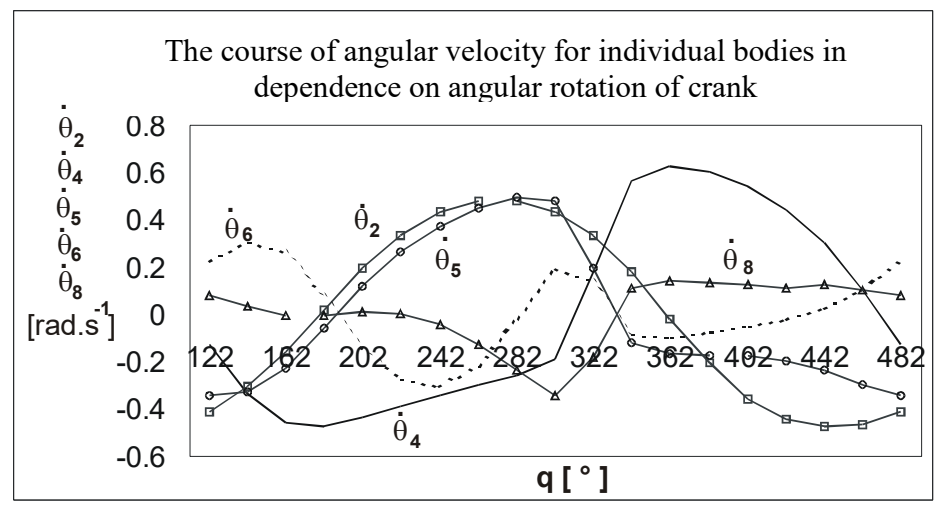

Fig. 7. The course of angular velocity for individual bodies in dependence on angular rotation of crank 


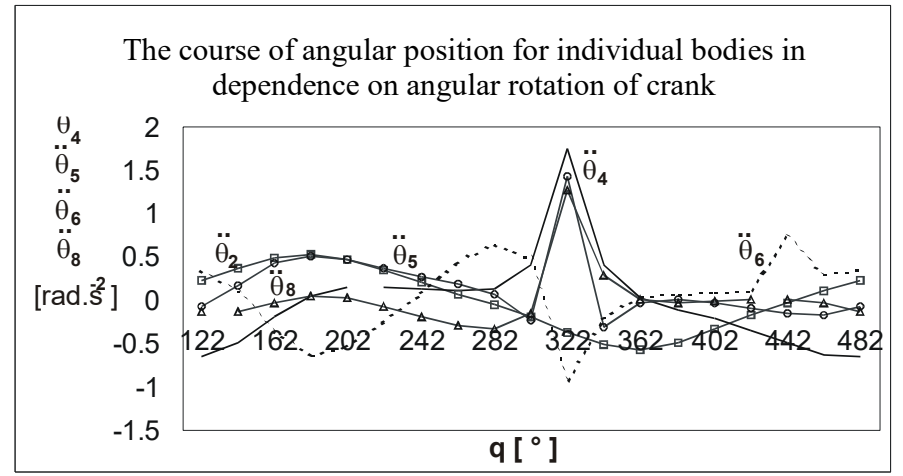

Fig. 8. The course of angular acceleration for individual bodies in dependence on angular rotation of crank

\section{Conclusion}

Based on the evaluation of the results, the utilisation of the Motion Program is significantly useful because it is effective way to determine all kinematic parameters of any mechanism and moreover, the loading for any point of the body system is able to be specified. The tolerance for the position deviation was also tested while the predetermined deviation was $10^{-9}$. It is important to point out that from the aspect of convergence, it was not necessary to use more than five steps for each one position. On the other side, the convergence failure was connected with specification and entering of inaccurate parameters.

This work was supported by the Slovak Grant Agency KEGA 007TnUAD-4/2017.

\section{References}

1. B. Paul, Kinematics and Dynamics of Planar Machinery, Prentice-Hall, New Jersey, (1979)

2. V. Brát, Handbook of kinematics with examples, SNTL, Prague, (1976)

3. A. Sapietová, M. Saga, B. Hyben, M. Sapieta, Effective methods of parameters refinement of machinery in the program MSC ADAMS, Applied Mechanics and Materials 611, 67-74 (2014)

4. A. Sapietová, M. Sapieta, B. Hyben, Document Sensitivity analysis application for multibody system synthesis, Applied Mechanics and Materials 420, 68-73 (2013)

5. A. Sapietová, M. Sága, A. Shimanovsky, M. Sapieta, Mobility of multibody systems in terms of their incorrectness, Communications 16(3A), 6-12 (2014)

6. A. Sapietová, V. Dekýš, M. Vaško, A numerical model of rotating machine having unbalance and the measurements of its dynamical properties, Metalurgija (Metalurgy) 49, 503-507 (2010)

7. Vavro, J. jr., Vavro, J., Kováčiková, P., Bezdedová, R., Kinematic and dynamic analysis of planar mechanisms by means of the SolidWorks software, Procedia Engineering 177, 476-481, (2017) 